\title{
Le multiculturalisme mondialisé: le défi de l'hétérogénéité
}

Jocelyne Cesari

\section{(2) OpenEdition \\ 1 Journals}

Édition électronique

URL : http://journals.openedition.org/conflits/153

DOI : $10.4000 /$ conflits. 153

ISSN : $1777-5345$

Éditeur :

CCLS - Centre d'études sur les conflits lilberté et sécurité, L'Harmattan

Édition imprimée

Date de publication : 15 mai 1999

ISSN : 1157-996X

Référence électronique

Jocelyne Cesari, «Le multiculturalisme mondialisé: le défi de l'hétérogénéité », Cultures \& Conflits [En

ligne], 33-34 | printemps-été 1999, mis en ligne le 16 mars 2006, consulté le 30 mars 2021. URL :

http://journals.openedition.org/conflits/153 ; DOI : https://doi.org/10.4000/conflits.153

Ce document a été généré automatiquement le 30 mars 2021.

Creative Commons License 


\title{
Le multiculturalisme mondialisé: le défi de l'hétérogénéité
}

\author{
Jocelyne Cesari
}

1 La mondialisation, ce maître mot désignant l'ouverture croissante des marchés nationaux aux exigences du commerce international est souvent définie comme l'étape ultime de l'avènement de l'économie de marché qu'on le déplore ou qu'on l'exalte ${ }^{1}$. Si ces processus sont désormais massivement étudiés, l'approche scientifique des dimensions culturelles de la mondialisation, quant à elle, n'en est qu'à ses débuts. Il est même regrettable que ce terme opère surtout comme cliché, sorte de "mot-valise " sollicité en dernière instance pour évoquer plus qu'expliquer les transformations en cours. La "Macdonaldisation» du monde est l'aspect le plus évident de cette mondialisation culturelle. Cette appellation désigne le processus multiforme d'homogénéisation, consistant en une mise en conformité des comportements culturels par rapport au canon que serait le modèle occidental. Cette homogénéisation recèle plusieurs aspects. Certes, la propagation des styles de vie, vêtements, musique, consommation venus de l'Occident est la plus visible et la plus frappante. Mais outre l'effet MacDonald et Coca-Cola, l'uniformisation progresse également à la faveur de la "culture Davos $»^{2}$ et plus subtilement encore avec la propagation d'un répertoire de normes et de valeurs tels que Droits de l'Homme, Démocratie, Economie de marché, Protection de l'environnement... s'imposant dans tous les points du globe. La culture Davos est la conséquence directe de la mondialisation économique et renvoie, non seulement à un style d'opérations économiques, mais aussi à un genre de vie. C'est pourquoi le "yuppie style » n'est pas qu'une formule : il correspond véritablement à des modes de consommation, de loisirs, de vie familiale engendrant toute une série de services annexes. Si les traits principaux de cet «art de vivre" sont occidentaux, ses hérauts et représentants proviennent de cultures et de sociétés très différentes ${ }^{3}$. Enfin, la culture de club est un autre aspect de l'homogénéisation culturelle porté par d'autres groupes sociaux et se définissant par une propagation des valeurs et idéologies occidentales. Par exemple, si les membres de la culture Davos vendent des ordinateurs dernier cri à la population indienne, les représentants de la culture de club, intellectuels, universitaires, journalistes, opérateurs culturels... promeuvent auprès de 
la même population, les vertus de la démocratie de marché, de la protection de l'environnement ou encore du féminisme dans sa version occidentale. Mais l'homogénéisation est loin de résumer le phénomène de mondialisation culturelle. Il n'est en effet plus possible de considérer que la mondialisation consistera principalement dans la croissance d'une culture unique en raison de la disjonction croissante entre l'économie, la culture et la politique, disjonction dont la théorisation en est à peine à ses débuts. Les images cohérentes et policées de la modernité projetées par l'Occident sont de plus en plus remises en cause et contestées par des formes culturelles alternatives. C'est pourquoi nous assistons à une augmentation des crises politiques liées aux conflits de valeurs, de normes et d'images. Mais si le conflit des valeurs est partout présent, il est quand même réducteur de considérer ces formes culturelles alternatives comme contre-modernité, c'est-à-dire uniquement réactionnelles et en tension avec l'Occident ${ }^{4}$. Cette dimension culturelle de la mondialisation oblige à reposer la question de la culture dans la théorie sociologique et notamment des relations entre culture et structure sociale ${ }^{5} \mathrm{La}$ difficulté réside dans le fait que cette question a longtemps été liée au débat marxiste et neo-marxiste à propos du développement du capitalisme ${ }^{6}$. La sociologie classique ayant en général adopté une approche nationale et stato-centrée, tout l'enjeu réside désormais dans la possibilité d'une production sociologique intégrant la dimension mondiale ${ }^{7}$. Dans cette perspective, les approches non réifiées de la société développées dans les travaux de Norbert Elias ou Georg Simmel offrent une piste heuristique dans la mesure où elles mettent l'accent sur les processus sociaux et les modes de sociation. Dans ces conditions, la mondialisation ne peut être envisagée sous la forme du tout ou rien : elle ne sera jamais ni complète désagrégation des systèmes sociaux existants ni complète intégration de ceux-ci dans une forme originale, homogène et cohérente. Dès lors, l'individu et non pas l'État-Nation tend à occuper une place centrale dans l'hétérogénéisation de la scène internationale ${ }^{8}$. Pour autant la scène internationale ne se dédouble pas entre d'un côté, le monde fini codifié et ritualisé de l'État et de l'autre, le monde polycentré et polyforme des acteurs non étatiques". Il est essentiel de reconnaître que l'action des acteurs sur la scène internationale est définie non plus par leur souveraineté ou leurs privilèges légaux mais par la nature des relations qu'ils tissent à l'échelle internationale. C'est pourquoi l'autorité et la souveraineté résident de plus en plus dans la capacité à influencer ou contrôler les ressources, les personnes et les enjeux par delà les territoires nationaux ${ }^{10}$. Cette dynamique complexe réfère à un triple mouvement de différentiation, relativisation et socialisation à l'échelle mondiale. La différentiation intensive des sociétés ne peut plus être pensée exclusivement dans le cadre des enceintes nationales. Longtemps associée à la diversification sociale et économique des sociétés nationales, elle est aujourd'hui davantage attachée à l'ethnicité et au multiculturalisme sur la scène mondiale. Les conflits dits interethniques sont dorénavant une dominante de la politique mondiale comme l'attestent d'ailleurs les troubles majeurs intervenus dans la dernière décennie de la guerre en Bosnie en passant par le Kosovo ou le Rwanda. La question simmelienne de l'étranger s'est intensifiée et a changé de nature dans la mesure où désormais même le voisin peut être un étranger à qui l'on n'est lié par aucune forme de considération ou de civilité. Le classique problème Hobbesien de l'ordre devient alors un problème mondial. La relativisation renvoie à la réflexivité, concept également utilisé par Anthony Giddens pour désigner l'institutionnalisation du doute comme pierre de touche de la modernité dans la mondialisation ${ }^{11}$. Cet affaiblissement de la croyance envers les institutions est 
certes un lieu commun de la littérature sociologique sur la modernité et la postmodernité. Plus rares sont ceux qui à l'instar de Roland Robertson mettent l'accent sur l'aspect mondialisé de ce doute. Tout l'enjeu est de savoir si cette ouverture infinie des possibles dans le domaine de la croyance et des valeurs recèle un risque de clôture ou en d'autres termes d'intégrisme ${ }^{12}$. Or il semble que le corollaire obligé de la relativisation et du doute généralisé soit justement l'exacerbation des tentations intolérantes. En d'autres termes, c'est parce que le choix des possibles en matière de normes et de croyances est de plus en plus grand que la réaction intégriste est aussi de plus en plus intense. Face à une telle fluidité des identifications et des allégeances, se pose alors la question du cadre institutionnel de la citoyenneté et des formes de socialisation. Si l'affaiblissement du cadre stato-national comme forme centrale et exclusive de la vie internationale est désormais largement tenu pour acquis et suscite de nombreux travaux, il n'en va pas de même en ce qui concerne l'engagement politique d'acteurs qui opèrent dans la dimension mondiale. Que sait-on des arrangements entre loyautés locales, nationales et internationales? Peuvent-elles réellement se combiner ou agissent-elles sur un mode antagonique ? En définitive, trois questions résument cette complexité sociologique liée à l'hétérogénéisation croissante de la scène internationale, auxquelles nous avons tenté de répondre dans ce numéro: est-ce que la société en tant que forme organisée des allégeances politiques et culturelles est encore possible dans un contexte de multiculturalisme mondialisé ? Estce que la stabilité du soi individuel est encore possible quand la réflexivité permanente est la conséquence de la relativisation mondiale? Comment la responsabilité et l'engagement individuels sont-ils mis en œuvre dans la société mondiale? Les marchands, immigrés, colporteurs, entrepreneurs, acteurs religieux, décrits dans ce numéro présentent cette double caractéristique d'être à la fois "transnationaux » et «indigènes ", en d'autres termes de s'inscrire dans l'hétérogénéisation de la scène internationale. Dans l'anonymat ils déploient au quotidien des formes d'action en s'appuyant sur des ressources culturelles qui ne sont pas celles de la culture Davos mais qui n'en sont pas moins efficaces. Eu égard à la fluidité et au caractère polymorphe des phénomènes transnationaux, ils font en quelque sorte partie de la face cachée de la mondialisation, plus difficile à investir que le monde des institutions. Ce dossier, «Les anonymes de la mondialisation », consacré à l'analyse théorique et méthodologique du transnational a été possible grâce à une recherche de cinq années dans l'aire méditerranéenne consacrée aux réseaux économiques entre l'Europe et le Maghreb ${ }^{13}$. Les contributions présentées ici sont issues d'un colloque qui s'est déroulé à Aix-enProvence en juillet 1998 afin de confronter les résultats obtenus en Méditerranée avec l'état des connaissances sur des phénomènes comparables dans d'autres aires géographiques et culturelles. La recherche sur les réseaux économiques entre l'Europe et le Maghreb s'inscrivait dans les orientations définies par la Politique méditerranéenne rénovée puis la Conférence euro-méditerranéenne de Barcelone en novembre 1995, privilégiant les nouvelles formes de coopération décentralisée et la prise en compte des acteurs non-institutionnels dans les échanges entre les deux rives. C'est pourquoi, cette étude, elle-même transnationale, a concerné les relations qui se nouent entre groupes installés de part et d'autre de la Méditerranée, c'est-à-dire entre individus originaires du Maghreb installés en Europe et ceux vivant au Maghreb, en considérant en quelque sorte cette dynamique d'échanges comme une coopération d'un genre nouveau fort éloignée de celle en vigueur entre États. En effet, dans les pays européens, qu'ils soient des pays d'immigration ancienne (comme la France) ou des 
pays d'immigration récente (comme l'Espagne ou l'Italie), ont émergé en provenance du Maghreb de nouvelles modalités de relations à l'espace européen. Si l'immigration de travail est officiellement arrêtée en Europe, il n'en demeure pas moins que des flux de circulation se maintiennent entre les deux rives de la Méditerranée : regroupement familial, entrées touristiques qui peuvent se prolonger, entrées à but économique, universitaire ou culturel. Ainsi, en Espagne comme en Italie, la majorité des nouveaux entrants en provenance du Maghreb, arrivés comme touristes ou étudiants, ont " prolongé » leur séjour et sont parvenus à obtenir du travail, la plupart du temps de manière non officielle. Par ailleurs, depuis une décennie, les centres urbains attirent pour des séjours de courte durée des individus en provenance du Maghreb et deviennent ainsi les lieux privilégiés de l'émergence de mouvements pendulaires entre les deux rives de la Méditerranée, au travers notamment d'activités économiques de consommation et d'achat, alimentant les secteurs de l'économie informelle au Maghreb. De tels flux d'échange supposent l'existence de liens et de réseaux entre les populations des deux rives. Cette nouvelle configuration des échanges contribue à l'émergence d'un espace médian entre Nord et Sud au sein duquel transitent, non seulement des hommes et des biens, mais aussi des idées, des valeurs politiques et culturelles. Cet espace médian n'est pas à proprement parler un territoire, il est construit par les mobilités d'un certain nombre d'individus, impliqués dans des activités dont l'efficacité est dépendante d'une transgression des frontières. Cette tendance à la transnationalisation des flux et des activités échappe en partie au contrôle des souverainetés étatiques des pays européens et des pays maghrébins et met en évidence l'autonomie des groupes sociaux dans le champ des relations internationales. Il en résulte des enjeux politiques, d'une part pour les États du sud de l'Europe, lesquels sont les plus exposés à ces flux et qui, dans le cadre de la construction européenne, ont comme fonction de garantir l'étanchéité de la frontière commune face aux pays d'Afrique, d'autre part, pour les États maghrébins qui peuvent essayer de réguler ces flux ou au contraire les utiliser comme «monnaie d'échange » et moyen de négociations dans leurs relations bilatérales avec les États européens comme dans leurs relation à l'Europe en construction. Il s'agissait donc d'appréhender concrètement les expressions de l'extra-territorialité de certaines formes d'activité économiques et culturelles qui affectent aujourd'hui la construction des identités politiques et rendent plus complexes les formes de loyauté. Grâce au colloque de juillet 1998, la comparaison avec d'autres groupes et d'autres espaces culturels s'est avérée essentielle. Les contributions présentées ici s'appuient sur une méthodologie commune focalisant sur la description des réseaux. Elles ont également permis d'éclairer sous un jour nouveau les nouvelles formes de capitalisme engendrées par la mondialisation et de rendre plus complexe la dialectique de l'État et du transnational.

2 Des réseaux d'entrepreneurs L'utilisation du terme "réseau», d'un maniement théorique et surtout méthodologique malaisé, apparaît cependant commode pour penser les relations transversales entre individus traversant les limites des organisations institutionnelles (collectivité nationale, locale). Ces relations ne sont pas par définition toujours dénombrables parce que fluctuantes et toujours susceptibles de s'étendre. Ce terme emprunté à la théorie des sciences sociales ${ }^{14}$ désigne toutes sortes de «mouvements faiblement institutionnalisés réunissant des individus et des groupes dans une association dont les termes sont variables et sujets à une interprétation en fonction des contraintes qui pèsent sur leurs actions $»^{15}$. L'approche en termes de réseau permet de montrer comment des espaces de mobilisation sont construits par 
delà les frontières par des acteurs privés, porteurs de ressources mais aussi de valeurs et de pratiques novatrices. Ceci est possible en raison du faible degré de formalisation et de coordination des relations et d'une absence de véritable spécialisation des rôles dans un système qui rassemble différents types d'acteurs sans poser de hiérarchie effective. Il en résulte une plurifonctionnalité des réseaux qui leur confère ce que d'aucuns appellent une multiplexité ${ }^{16}$. Une telle perspective permet de dépasser la frontière artificielle entre processus interne et externe dans l'observation de la dynamique sociale ou encore de combiner l'analyse des relations internationales avec celle de la politique comparée ${ }^{17}$. Le réseau en tant que construit sociologique permet de restituer la complexité des interactions entre l'individu et la structure en privilégiant la fluidité des intérêts et des identités. Pour ce faire, il est nécessaire d'associer l'étude des mouvements sociaux à celle de l'analyse constructiviste des Relations Internationales. La recherche peut ainsi être qualifiée de " grounded theory "; c'est-àdire une tentative pour parvenir à des approches théoriques à partir d'une approche empirique qualitative. Dès lors, l'objet de la recherche a été défini par l'analyse de ces relations transnationales entre rive Nord et Sud de la Méditerranée. L'échelle nationale n'est pas apparue comme la perspective la plus pertinente pour saisir l'organisation de ces réseaux. C'est pourquoi, leur déploiement a été mis en évidence à partir des espaces régionaux et urbains tant au Nord qu'au Sud. Ont été retenus : Marseille, Paris, Madrid, Barcelone, Ceuta et Melilla, Milan, Turin, Naples et Palerme au nord. Au Maghreb, ont été choisies des régions caractérisées par une forte tradition migratoire vers l'Europe et au sein desquelles les immigrés tendent à s'affirmer comme des acteurs économiques : la région du Souss, du Rif et du Tedla pour le Maroc, les régions de Médenine et Tataouine pour la Tunisie. C'est pourquoi, la question de l'ancrage des réseaux a été déterminante ${ }^{18}$. Les différents membres de l'équipe ont appréhendé, chacun depuis leur site d'observation, à partir de quelles organisations et de quels individus, les réseaux économiques se déploient dans un espace transnational. Tous ont répondu à la triple question emboîtée : qui fait quoi ? où ? ou plus précisément encore : qui exploite l'écart entre ici et là-bas et comment? La recherche a permis de dégager d'une part, des figures d'opérateurs créant ou réorientant à leur profit les ressources économiques transnationales et d'autre part, des positionnements géographiques qui deviennent des ressources dans la gestion du transnational. Donc l'acteur est déterminant du point de vue de l'analyse du réseau et dans ce cas l'accent a été mis sur deux sortes d'entrepreneur privé. Le premier prend place dans le milieu immigré vivant en Europe et présente différents profils selon que l'immigration est ancienne ou récente. Du côté maghrébin, la recherche a porté sur des groupes ethniques ou régionaux qui ont utilisé leur positionnement au cœur des circulations migratoires internationales comme une ressource dans le secteur de l'économie de marché ${ }^{19}$. Enfin, les réseaux transnationaux se donnent à voir dans les activités plus familières des activités transfrontalières. Lorsqu'elles consacrent, comme c'est le cas entre l'Europe et le Maghreb, une relation d'échange inégale, elles favorisent du même coup la recherche de plus-values en rentabilisant l'écart différentiel entre ici et là-bas : ce qui est petit «ici » est " grand » là-bas. C'est particulièrement vrai pour les nouvelles formes de mobilité du Maghreb vers l'Italie ou l'Espagne. De même les activités de contrebande entre Ceuta et Melilla et son arrière-pays marocain, ou encore le déploiement de l'économie de bazar à Marseille décrit par Michel Peraldi, illustrent de manière éclatante cet usage de la frontière comme ressource économique et culturelle. C'est pourquoi, la plupart des contributeurs, en investissant les réseaux, ont travaillé en fait sur la tension qui surgit 
au croisement de certaines activités et de certains lieux. Dans cette perspective, l'espace urbain est par définition un endroit privilégié pour la mise en évidence des réseaux transnationaux. Défini comme un ensemble de nœuds de communication, sa caractéristique est de rendre possible la coexistence de formes de capitalisme et de cultures fort différenciées et parfois présentées comme antagonistes comme le montre l'article de Saskia Sassen. Dans cette perspective, la proximité entre grandes métropoles économiques et culturelles comme Marseille, Paris, Madrid, Barcelone, Turin, Tunis, Alger, Casablanca est dans certains secteurs plus grande que celle existant entre ces centres urbains et leur arrière-pays respectifs.

Weber revisité Le capitalisme ne progresse pas en détruisant les particularismes mais au contraire en les instrumentalisant. A cet égard, la question weberienne de l'influence des mentalités et donc des cultures dans la dynamique de l'esprit capitaliste est apparue centrale dans les enquêtes. Or, la tentation est grande d'attendre des acteurs « non occidentaux » des comportements similaires à ceux du bourgeois puritain $\mathrm{du}$ XVIIIème siècle et de mesurer l'efficacité capitaliste au processus d'uniformisation des sociétés et de désenchantement $d u$ monde ${ }^{20}$. Ces entrepreneurs transnationaux mettent en évidence ces nouvelles formes d'accumulation et obligent à reposer la question weberienne de l'esprit du capitalisme. L'extension du capitalisme équivaut à sa réinvention par multiplication des différences. Les cas décrits dans l'enquête ou présentés dans le numéro, qu'il s'agisse des entrepreneurs djerbiens ou d'Europe de l'Est ou encore des chinois d'outre-mer, montrent que l'esprit schumpetérien s'accommode fort bien et même tire profit des liens de parenté, de l'esprit communautaire, en bref de ce que Weber n'hésitait pas à qualifier à propos de la Chine " d'entraves claniques à l'économie ». A cet égard, l'opposition classique entre réseaux organiques qui définissent le tissu des relations au sein d'une communauté et recouvrent diverses fonctions et réseaux fonctionnels ou stratégiques créés à de fins précises et délimitées tend à devenir inopérante ${ }^{21}$. Ces réseaux révèlent tout à la fois de nouveaux usages du territoire et de l'ethnicité en jouant sur une dialectique incessante entre le local et l'international. Le point de vue constructiviste adopté par l'ensemble des contributeurs permet de capter «sur le vif » les rapports subtils entre le groupe et l'individu à travers le déploiement d'une activité économique qui s'étend sur plusieurs territoires nationaux et active des ressources propres au groupe que celles-ci soient d'ordre culturel, géographique ou contextuel comme par exemple le cas des entrepreneurs djerbiens décrits par Hassen Boubakri. Dans le milieu immigré vivant en Europe, apparaissent différents profils selon que l'immigration est ancienne ou récente. Les questions sur l'avènement de diasporas et l'émergence de l'ethnic business ont alors été centrales et ont permis de dépasser le dilemme classique : intégration dans le pays d'accueil/retour au pays d'origine. En effet, l'installation en Europe ne signifie plus rupture définitive avec le pays d'origine, bien au contraire. On assiste à des déploiements d'activités qui se jouent des frontières et qui du Nord au Sud de la Méditerranée opèrent du local à l'international. C'est particulièrement vrai dans le cas des individus ayant créé des entreprises. C'est la combinaison de facteurs externes (crise de l'emploi en Europe, évolution de l'appareil commercial et des modes de consommation) et de facteurs internes (tradition migratoire et commerçante, structures de solidarité, développement d'une demande immigrée) qui expliquent l'essor du commerce et des entreprises tenues par des étrangers. Mais il faut insérer ces stratégies entrepreneuriales dans d'autres plus globales qui concernent l'autonomie du groupe ethnique. A ce propos, le partage de valeurs liées à une origine commune va 
s'inscrire dans l'ensemble des relations sociales et favoriser les opportunités économiques avec le Maghreb. Dans le cas d'immigration récente comme en Espagne ou en Italie, l'enclave ethnique est la forme dominante (à la différence des pays d'immigration ancienne où les activités économiques indépendantes sont en cours de diversification). Par enclave ethnique, il faut comprendre visibilité et consolidation dans un espace urbain d'activités gérées par des entrepreneurs migrants. Deux conditions président à la création et au maintien de ces activités : l'existence d'un besoin et de moyens financiers dans le groupe et l'arrivée permanente de personnes. C'est bien ce qui est en train de se produire dans certains grands centres urbains comme Madrid, Barcelone mais aussi Milan, Turin et Palerme où ont été recensés des commerces de produits ethniques (viande halal, épices) liés à l'arrivée récente des gens originaires du Maghreb. A ce propos, il a été intéressant de constater que les relations avec le pays d'origine sont complètement partie prenante du processus d'intégration dans les nouveaux pays d'accueil. Cette approche des actions économiques et la mise en place de réseaux ethniques transnationaux oblige à poser la question des valeurs, des loyautés et donc des formes d'identité situées au cœur de ces actions. Dès lors, peut-on définir comme diaspora toute forme de groupe ethnique à dimension transnationale ? En d'autres termes, le fait d'exister à une échelle internationale sous la forme de l'exil, de la dispersion et du maintien d'une conscience commune, légitime-t-il l'usage du terme diaspora? Si l'on considère que la condition de diaspora engendre une culture spécifique distincte de celle du lieu d'origine comme l'article d'Emmanuel Ma Mung tend à le démontrer dans le cas des "Chinois d'Outre-mer ", tel n'est pas le cas des groupes issus du Maghreb. La polarité se joue encore presque exclusivement dans le rapport au pays d'origine, particulièrement dans la gestion des activités économiques ou sociales ${ }^{22}$. En définitive, les différentes approches et notamment celles d'Emmanuel Ma Mung ou encore de Valérie Amiraux sur l'islam turc, mettent en lumière les mécanismes concrets de déterritorialisation des activités économiques ou politiques et des identités en montrant la relation dialectique et complexe existant entre ces deux processus. Ainsi, l'approche par les réseaux permet de déceler les circulations et mobilités des immigrés/émigrés en présentant comme un seul système d'action cohérent leur positionnement dans les sociétés européennes et leur insertion dans les espaces maghrébins. C'est ainsi que d'un côté, ils sont en Europe des entrepreneurs maghrébins sans que soit vraiment perceptible la dimension transnationale d'une certaine forme d'ethnicité qui leur permet de bénéficier de ressources supplémentaires dans l'action économique. En fonction de chaque groupe et du contexte dans lequel il se déploie, il est ainsi possible d'établir un répertoire des ressources utilisées. Dans les espaces maghrébins, ces acteurs ont un rôle et une fonction non négligeable dans les espaces locaux, dans la mesure où les bénéfices retirés de leur positionnement transnational sont réinvestis à la fois dans l'espace économique social mais aussi politique de la région d'origine. Du côté maghrébin, la recherche avait porté sur des groupes ethniques ou régionaux qui ont utilisé leur positionnement au cœur des circulations migratoires internationales comme une ressource dans le secteur de l'économie de marché. Les tunisiens issus de Djerba et de Ghomrassen sont en train de déployer de véritables entreprises transnationales sur les deux rives en utilisant les réseaux familiaux et un savoir-faire initial auquel ils ont intégré de nouvelles activités et donc de nouvelles compétences (restauration, hôtellerie, textile, électronique) à la fois en Tunisie et dans plusieurs centres urbains européens et même en Asie! Ces formes réinventées, bricolées de l'activité capitaliste sont également liées au 
télescopage du temps mondial avec les temps nationaux et régionaux qui place les lieux d'où partent les réseaux au centre des dynamiques migratoires entre l'Europe et le Maghreb : c'est vrai pour le développement de la région du Souss au Maroc comme pour celle du Tedla, devenue très récemment terre d'émigration vers l'Italie. A cet égard, la dynamique historique longue entre l'Europe et le Maghreb ainsi que les enjeux de proximité ne doivent pas être négligés comme ressources dans l'activité économique transnationale de ces entrepreneurs. La mobilité permanente remplace les formes traditionnelles de migration y compris dans des pays d'immigration ancienne comme le Maroc et la Tunisie qui se mettent ainsi au diapason des nouvelles formes des mobilités pendulaires incessantes entre espaces du pays d'origine et différents lieux de destination telles que les décrit Mirjana Morokvasic-Muller dans ce numéro à propos des migrants d'Europe de l'Est. L'émigration de la région du Souss au Maroc s'est toujours produite et développée dans le cadre d'un système complexe de relations et de réseaux de tout ordre. C'est pour cette raison que les mouvements migratoires, au départ de cette région, persistent encore sous de nouvelles formes, à tel point qu'ils sont devenus une donnée structurelle du paysage démographique et socio-économique régional. Il semble bien que le phénomène est en passe de se renforcer et de s'amplifier davantage à court et à moyen terme. Car, d'une part, l'émigration étrangère soussi n'est plus l'apanage des seuls démunis. Le caractère qu'elle revêt actuellement a offert l'opportunité par le biais de l'investissement et de l'entreprenariat, tels qu'ils se sont produits jusqu'à présent, pour que le phénomène s'élargisse et s'applique à d'autres couches de la société et plus particulièrement à la bourgeoisie. En ce sens, l'émigration n'est plus une affaire de départ d'hommes ou de main-d'œuvre, telle qu'elle se présentait jusqu'aux années 70, mais elle s'est également doublée d'un aspect non moins important, à savoir le départ de capitaux. D'autre part, l'émigration, du fait de ses impacts multiples, a largement contribué à l'extraversion de la société et à l'ouverture de toutes ses composantes régionales sur l'étranger, lequel étranger est devenu ainsi dans le fait, comme dans l'inconscient des populations, toutes catégories d'âge confondues, une portion indissociable du territoire ou de l'espace de vie. De même, la notion de bled commence à revêtir une connotation nouvelle qui s'approche plus de celle d'espace de vie que de celle du pays d'origine. Le Souss se dégage comme s'il s'agit d'un territoire qui s'étend, sous formes d'auréoles, de Guelmim jusqu'à l'Europe du Nord, et ce en fonction de l'importance de la présence soussie le long de cet itinéraire. Dans le Rif oriental, l'esprit d'entrepreneuriat des populations conjugué aux difficultés de la région, a porté les éléments les plus dynamiques vers d'autres horizons. Cependant bien que répulsive, la région retient ces « fils » qui, malgré l'éloignement, tiennent absolument à maintenir des relations étroites avec leur lieu de naissance, relations qui se traduisent par la création d'entreprises sur place. Mais à côté de cette émigration de pauvreté, existe une émigration de personnes ne manquant de rien mais cherchant l'ouverture sur l'extérieur. La présence d'une frontière et d'une tradition de commerce parallèle qui remonte à l'époque des deux protectorats français et espagnol, accuse cette extraversion qui débouche sur une territorialité morcelée et des individus qui s'identifient à des espaces multiples selon un système d'auréoles ayant comme centre de gravité Nador. Dotée de ressources plus que suffisantes et éloignée de toute frontière et de toute tentation de trafic illicite, la plaine du Tadla a également été touchée par les mêmes phénomènes et l'installation de flux migratoires qu'appuient des flux de biens et d'argent. Ainsi les relations avec l'Italie sont devenues une composante structurelle du tissu économique, culturel et social de cette région 
agricole. La fermeture des frontières des pays européens incite ceux désireux de lutter contre le chômage par le biais de l'émigration internationale, à se rabattre sur des formules qui permettent l'ouverture sur l'extérieur, d'où les nombreux entrepreneurs qui utilisent le rachat ou l'héritage de petites entreprises nées de l'émigration dans des pays européens. Des notions comme l'entreprenariat économique, le libre échange entre les pays riches de la rive nord et les pays pauvres de la rive sud, le partenariat en matière de formation et de recherche scientifique, l'Université Méditerranéenne itinérante, etc.., sont autant de formules et de concepts illustrant les préoccupations parfois toutes formelles d'un Nord riche à l'égard d'une rive sud méditerranéenne qui serait caractérisée par le sous-développement. Parallèlement à ce discours officiel qui accompagne le phénomène de mondialisation, il y a en fait la mise en place d'une réalité informelle mais réelle que les travaux sur les réseaux transnationaux entre l'Europe et le Maghreb ont contribué a mettre au jour et qui est loin d'être prise en compte dans les recensions économiques ou sociales de la technocratie bruxelloise à propos de l'aire méditerranéenne.

Dialectique de l'État et des réseaux : contestation perturbation, régulation Il ne faudrait pas en déduire trop rapidement une "abolition de la géographie" ni même du territoire. Le transnational se nourrit du territoire : à cet égard, le phénomène de mondialisation conduit trop souvent à des diagnostics pessimistes sur la fin inéluctable des États ${ }^{23}$. En revanche, l'aspect de la mondialisation qui renvoie à la progression accélérée des techniques de communication et de circulation a introduit un changement, non seulement quantitatif, mais aussi qualitatif dans la gestion étatique. A une territorialité faite de bornage et de souveraineté a succédé une autre géographie faite de coalitions changeantes et de réseaux. L'organisation de l'espace ne correspond plus aux données et est prioritaire dans l'allocation des ressources et la diffusion des valeurs. Les États ont de moins en moins le contrôle de l'évolution économique et financière mais ils sont encore tenus pour politiquement responsables par leur population. Les nouvelles formes d'identification contribuent à la multiplication des loyautés qui peuvent venir contrarier l'allégeance citoyenne et du même coup rendre plus difficile la construction du pacte social toujours recherché sur des bases nationales alors que les contraintes et les mobilités sont dorénavant mondiales. Pour autant une dialectique subtile s'installe entre États et réseaux qui ne se réduit pas à la tension. A cet égard, l'article de Valérie Amiraux sur l'organisation de l'islam entre Allemagne et Turquie vient éclairer cette subtile interaction entre réseaux de l'islam immigré et État turc ou allemand. De même dans le domaine économique, la recherche sur les réseaux entre l'Europe et le Maghreb a montré que la privatisation et le passage à l'économie de marché ne signifient pas toujours désengagement de l'État. En même temps, évaluer la seule dimension macro-économique de la mondialisation qui peut le plus souvent conduire à des bilans négatifs, ne permet pas de comprendre les apports plus modestes mais en tout cas régulateurs des réseaux transnationaux à l'échelle micro-économique. En d'autres termes, le transnational sert l'État et l'État sert le transnational et il est bon de mesurer les procédés et les circonstances d'une telle "collaboration». Cette dialectique est perceptible dans les conséquences économiques de ces réseaux sur les économies maghrébines, qu'il s'agisse des changements macro-économiques ou bien de l'émergence de nouvelles catégories d'entrepreneurs privés en lien avec l'économie de marché. Enfin, les mouvements transfrontaliers comme ceux observés dans le cas de Ceuta et Melilla révèlent à quel point les phénomènes transnationaux sont d'une certaine manière dépendants de l'existence de frontières et donc de bornages étatiques. 
De tels réseaux transnationaux apparaissent comme des facteurs de changement économique et social au Maghreb. A cet égard, la catégorie des entrepreneurs privés, en partie engendrée par les Plans d'Ajustement structurel a été privilégiée, notamment ceux qui ont construit ou construisent une relation économique avec le sud de l'Europe même s'ils n'ont pas d'expérience migratoire. La dimension transnationale va se présenter sous deux aspects : d'une part, lorsque les entrepreneurs sont engagés dans des activités nécessitant la mobilisation de ressources extérieures (sociétés mixtes, activités d'import/export), d'autre part, lorsque l'État lui-même est soumis aux contraintes internationales. Les enquêtes dans le secteur privé ont révélé l'influence des réseaux transnationaux dans les transformations économiques en cours, particulièrement en Algérie où l'économie est devenue en grande partie « extravertie ». Cette externalisation concerne, non seulement les opérateurs privés, mais bien évidemment l'État lui-même comme l'atteste l'étude de cas de la politique économique tunisienne. Ce dernier aspect ne correspond pas à une défaite de l'État ni même à l'apparition d'un État « circonscrit ». En fait la compétition internationale, voire même la mise en tutelle par le biais des Plans d'Ajustement Structurel se traduit par la création de nouvelles fonction étatiques (politiques de subvention aux exportations, soutien des "champions nationaux" de la privatisation..) et de nouveaux rapports entre État et société civile, comme le montre le cas marocain. Nous sommes ainsi confrontés au paradoxe suivant: le désengagement de l'État dans le domaine économique s'accompagne bien souvent d'un renforcement de ses modalités d'intervention politique.

5 L'utopie de la société civile internationale En conclusion, ces réseaux transnationaux consacrent le processus d'individualisation en cours de part et d'autre de la Méditerranée, même lorsque les liens de parenté ou la logique du groupe ethnique semblent s'exercer de manière forte. Il faut donc sortir des perceptions trop « occidentalo-centrées » de la modernité et de l'efficacité économique afin de restituer à leur juste valeur les comportements et logiques à l'œuvre y compris dans ce qui paraît le plus anodin ou le plus insignifiant au regard de données macrocospiques. Ils mettent également en évidence la construction d'espaces d'action et de référence qui combinent en même temps, le niveau local, national et international. Lorsque de surcroît, les acteurs sont non étatiques, toutes les conditions de la gouvernance sont réunies, procédure qui tend à associer les interactions entre acteurs publics et privés dans la confection des politiques publiques. A cet égard, les modalités de la coopération décentralisée, lorsque leur objectif est de favoriser du partenariat entre des acteurs issus des sociétés civiles de part et d'autre de la Méditerranée correspond à ce principe. Enfin, la multiplication de ces réseaux transnationaux est l'indice d'appartenances multiples qui dépassent le cadre stato-national et mettent en connexion des espaces de culture et d'histoire différentes. C'est pourquoi, du côté européen, l'équilibre entre altérité et civilité est devenu aujourd'hui le problème fondamental des systèmes politiques contemporains. En ce qui concerne les États au Maghreb, ils se trouvent confrontés, comme tous les États, à une contradiction entre d'une part, la transnationalisation croissante des échanges économiques et d'autre part, des affirmations nationales maintenues. La situation des diasporas est au cœur de cette contradiction. Elles mettent en lumière deux aspects significatifs de cette contradiction : leur existence même révèle le cadre contraignant dans lequel s'inscrit la politique européenne des États maghrébins, mais peuvent toutefois être envisagées comme un atout dans l'élaboration de cette politique comme dans le cadre des relations 
bilatérales. C'est pourquoi, le souci de préserver des liens avec les populations sédentarisées en France et en Europe implique également la volonté de tenter de définir une politique commune envers ces populations, notamment dans la perspective de l'Union Européenne. A cet égard, on constate une modification sensible de l'attitude des dirigeants maghrébins par rapport à leurs ex-ressortissants installés en Europe. Certains milieux sociaux et culturels sont plus impliqués que d'autres dans ces activités transnationales (entrepreneurs privés, jeunesse urbaine, intellectuels et enseignants), cette "extraversion" ayant des conséquences sur les changements économiques et sociaux en cours au Maghreb. A cet égard, la catégorie des entrepreneurs privés est très révélatrice, notamment avec ceux qui ont construit ou construisent une relation économique avec l'Europe. Se posait alors la question de savoir si certains de ces acteurs n'étaient pas d'ores et déjà engagés dans une sorte de société civile transnationale faisant voler en éclats les repères territoriaux des identités citoyennes, ce qui n'est pas sans conséquence au regard des transformations politiques et culturelles et des remises en cause que traverse actuellement le Maghreb. Il est possible d'affirmer que la réponse est pour l'instant négative: l'investissement dans le transnational favorisant davantage des attitudes d'exit et de repli, voire d'indifférence par rapport à la sphère du politique, et ce qu'elle se situe de part ou d'autre de la Méditerranée. Plus généralement, il est utopique de penser que la diffusion de formes culturelles mondiales a comme corollaire l'édification d'une civilité transnationale. Il est plus vraisemblable de penser en termes de conflits, fragmentation et contestation politique dans l'arène mondiale. Dès lors, la question pertinente renvoie à la manière dont certains groupes et certaines idées émergent et sont légitimés à l'échelle internationale ${ }^{24}$.

\section{NOTES}

1. Elle correspond à un démantèlement continu des territoires des États-nations par les réseaux financiers, sans cesse activés par les mesures de dérégulation qui ne font que renforcer la fluidité des capitaux. L'entreprise elle-même s'inscrit dans cette nouvelle pratique de l'espace : production, consommation, méthodes de gestion, comportements et valeurs des acteurs, tout obéit de plus en plus à des logiques qui transgressent les frontières comme en témoigne par exemple l'amplification des délocalisations. 2. Du nom de ce sommet économique qui réunit annuellement dans une luxueuse station suisse les principaux acteurs et opérateurs économiques du monde.

3. A tel point qu'un nouveau marché s'est développé, spécialisé dans l'inculcation de cette culture spécifique dans l'entreprise, suscitant l'émergence de véritables agents de socialisation à l'échelle internationale. Voir Berger Peter, « Four Faces of Globalization ", The National Interest, $n^{\circ} 49$, automne 1997.

4. Cf. Meyer J. W., «The World Polity and the Authority of the Nation-State » in Bergesen A. (ed.), Studies of the Modern World System, New York, Academic, 1980, pp. 109-137.

5. Voir Robertson Roland, Globalization, Social Theory and Global Culture, Sage, 1992. 
6. En dehors de l'approche marxiste classique de l'impérialisme, voir notamment les travaux d'Immanuel Wallerstein, The Modern World System 1, NewYork, Academic Press, 1974 ; The Modern World System 2, New York, Academic Press, 1980.

7. A cet égard, la tentative de Robertson n'est pas totalement convaincante dans la mesure où même sa critique de la sociologie classique oscille entre l'hommage à certains pères fondateurs (Weber notamment) et la remise en cause de ces mêmes travaux, Robertson R., op. cit.

8. Voir Turner Bryan S., Orientalism, Postmodernism and Globalization, Routledge, 1994.

9. Rosenau James, Turbulence in Worl Politics, Princeton, Princeton University Press, 1990.

10. Ferguson Yale H., Mansbach Richard W., « The Past as Prelude to the Future? Identities and Loyalties in Global Politics » in Lapid Yosef, Kratochwill Friedrich (eds.), The Return of Culture and Identity in IR Theory, Lynne Rienner Publishers, 1997, pp. 21-44.

11. Giddens Anthony, Modernity and Self-Identity : Self and Society in the Late Modern Age, Cambridge Polity Press, 1991.

12. L'intégrisme peut en effet être défini comme une clôture de la pensée, religieuse ou pas, consistant à rejeter les opinions ou croyances en contradiction avec ses propres convictions voir Cesari J., « Intégrisme », Dictionnaire des idées rebelles, Larousse, Paris, 1998.

13. Voir Cesari (J), Réseaux transnationaux entre l'Europe et le Maghreb, Commission Européenne ; Bruxelles ; 1997, 2 volumes. Je voudrais ici saluer les apports de tous les membres de cette équipe de recherche plurinationale pendant plus de cinq années, lesquels n'ont pu être restitués dans ce numéro. L'équipe française : Sabah Chaib (IEP Paris). L'équipe espagnole : Bernabé Lopez-Garcia, Professeur à l'Université autonome de Madrid, Anna-Isabel Planet (doctorante à l'UAM), Jordi Moreras (Barcelone, Cibedo), Encarna Cabello. L'équipe italienne : Jean-Pierre Cassarino (Institut européen de Florence), Ottavia Schmidt de Friberg (Université de Padoue). L'équipe algérienne : Abdelwahab Rezig, Slimane Bedrani, Ahmed Bouyacoub (Université d'Alger). L'équipe tunisienne : Hassan Boubakri (Université de Sousse), Mostefa Kharoufi (Ministère de la Jeunesse, Rabat), Aïcha Korched-Ettayeb (Université de Sousse). L'équipe marocaine : Mohamed Berriane, Professeur de géographie à l'Université Mohamed V de Rabat, Hnaka Othmane (Université d'Agadir), Salah Mounir (Université de Rabat). Je voudrais aussi remercier tous les participants au colloque de juillet 1998 et notamment : Valérie Angles-Hao (IRSEA, Aix-en-Provence), Joachim Arango ( Universitad Complutense, Madrid), Barabara Baille (IRSEA, Aix-en-Provence), Myriam Catusse, (IEP, Aix-enPorvence), Gaia Danèse (IEP, Paris), Rémy Leveau, (IEP Paris), Abdel Kader Sid-Ahmed (IEDES, Pais), Stéphane de Tapia (Migrinter, Strasbourg), Lionel Urdy (Université de la Méditerranée), Catherine de Wenden, (CERI), Aristide Zolberg,(Center for Migration, Ethnicity and Citizenship, New York).

14. Voir Granovetter Mark, «The strength of Weak ties : a Network Theory Revisited » in Marsden Peter. V., Lin Nan (eds.), Social structure and network analysis, London, Sage publication, 1982, pp. 105-130.

15. Voir Colonomos Ariel, «Sociologie et science politique : les réseaux, théorie et objet d'études », Revue Française de Science Politique, n 1, février 1995, pp. 165-178.

16. Voir Degenne Alain, Forsé Michel, Les réseaux sociaux, Paris, Armand Colin, 1994. 
17. Keck Margaret E., Sikkink Kathrin, Activists beyond Borders, Advocacy Networks in International Politics, Cornell University Press, 1998.

18. Voir Schijf (Huibert), Fennema (Meindert), « The transnational network » in Stokaman Frans, Ziegler Rolf, Scott John (eds.), Networks of Corporate Power, a Comparative Analysis of ten Countries, Cambridge, Polity Press, 1985.

19. Cette présentation des réseaux selon leur ancrage dans les sociétés du nord ou du sud de la Méditerranée a été adoptée pour les besoins de l'analyse mais ne rend pas compte de la fluidité des liens qui se répartissent en même temps sur les deux rives. 20. Pour une approche des nouvelles formes de capitalisme, voir Bayart Jean-François (dir.), La réinvention du capitalisme, Paris, Karthala, 1994. Berger Brigitte et alii, Esprit d'entreprise, cultures et sociétés, Paris, Maxima, 1993 (tr. Fr.).

21. Voir Godsell Gillian, « Entrepreneurs en état de guerre : l'entreprise en Afrique du sud » in Beger B., op. cit., pp 37-52.

22. Nos propres travaux en cours sur l'islam transnational tendent à montrer que cette construction d'une culture spécifique est en cours dans certains groupes musulmans qui parviennent à déterritorialiser et dénationaliser la question islamique. Voir Cesari J., Musulmans et Républicains, les jeunes, l'islam et la France, Bruxelles, Complexe, 1998.

23. Paul Hirst, Graham Thompson, Globalization in question, Blackwell, London, 1996. Selon ces auteurs, les économies ne sont pas plus ouvertes aujourd'hui qu'elles ne l'étaient avant 1914.

24. Hurrel Andrew, Woods Ngaire, « Globalization and Inequality », Millenium, 24, 3, 1998. Bull Hedley, The Anarchical Society : A Study of Order in World Politics, New York, Columbia University Press, 1995, 2ème ed.

INDEX

Mots-clés : Mondialisation, identité

\section{AUTEUR}

JOCELYNE CESARI

Chargée de recherches au CNRS-GSRL. 